\title{
ARTICLE Prophylactic ketamine alters nucleotide and neurotransmitter metabolism in brain and plasma following stress
}

Josephine C. McGowan ${ }^{1}$, Collin Hill ${ }^{2}$, Alessia Mastrodonato ${ }^{3,4}$, Christina T. LaGamma ${ }^{4}$, Alexander Kitayev ${ }^{2}$, Rebecca A. Brachman ${ }^{3}$, Niven R. Narain ${ }^{2}$, Michael A. Kiebish ${ }^{2}$ and Christine A. Denny ${ }^{3,4}$

Recently, we have shown that ketamine given prior to stress exposure protects against the development of depressive-like behavior in mice. These data suggest that it may be possible to prevent the induction of affective disorders before they develop by administering prophylactic pharmaceuticals, a relatively nascent and unexplored strategy for psychiatry. Here, we performed metabolomics analysis of brain and plasma following prophylactic ketamine treatment in order to identify markers of stress resilience enhancement. We administered prophylactic ketamine in mice to buffer against fear expression. Following behavioral analyses, untargeted metabolomic profiling was performed on both hemispheres of the prefrontal cortex (PFC) and the hippocampus (HPC), and plasma. We found that prophylactic ketamine attenuated learned fear. Eight metabolites were changed in the PFC and HPC upon ketamine treatment. Purine and pyrimidine metabolism were most significantly changed in the HPC, PFC, and, interestingly, plasma of mice two weeks after prophylactic administration. Moreover, most precursors to inhibitory neurotransmitters were increased whereas precursors to excitatory neurotransmitters were decreased. Strikingly, these long-term metabolomic changes were not observed when no stressor was administered. Our results suggest that prophylactic treatment differentially affects purine and pyrimidine metabolism and neurotransmission in brain and plasma following stress, which may underlie the long-lasting resilience to stress induced by a single injection of ketamine. These data may provide novel targets for prophylactic development, and indicate an interaction effect of prophylactic ketamine and stress. To our knowledge, this is the first study that identifies metabolomic alterations and biomarker candidates for prophylactic ketamine efficacy in mice.

Neuropsychopharmacology (2018) 43:1813-1821; https://doi.org/10.1038/s41386-018-0043-7

\section{INTRODUCTION}

Ketamine, a non-selective glutamate $N$-methyl-D-aspartate (NMDA) antagonist, has been shown to have rapid-acting antidepressant effects in treatment-resistant major depressive disorder (MDD) (TRD) patients [1]. The antidepressant onset is typically within $2 \mathrm{~h}$ and these effects persist for 1-2 weeks following a single infusion $[2,3]$. In addition to having efficacy in MDD, ketamine has therapeutic effects for posttraumatic stress disorder (PTSD) [4], suicide ideation [5], bipolar depression [6, 7], and anxious bipolar depression [8].

Rather than treating symptomatology in psychiatric disorders, a novel approach has recently been suggested to prevent the induction of psychiatric disorders before they develop by administering prophylactic pharmaceuticals. It has been proposed that it may be possible to take prevention approaches, including pharmacological ones, for PTSD [9]. One study administered the kappa-opioid receptor (KOR) antagonist nor-binaltorphimine (norBNI) before the fear-potentiated startle (FPS) test and found that it attenuated conditioned fear $[10,11]$. More recently, we found that a single injection of ketamine $\left(30 \mathrm{mg} \mathrm{kg}^{-1}\right) 1$ week before a stressor prevented stress-induced depressive-like behavior and social avoidance behavior for up to 4 weeks after injection [12]. Ketamine was efficacious as a prophylactic in three mouse models of stress, including learned helplessness (LH), social defeat (SD), and chronic corticosterone (CORT) administration. This efficacy was confirmed using a LH model in rats [13] and a chronic CORT model in mice [14]. Interestingly, the time window for prophylactic efficacy was limited to approximately 1 week before a stressor, but not 1 month or $1 \mathrm{~h}$ before [15].

To date, the mechanisms underlying prophylactic efficacy have yet to be identified. One potential area of interest is metabolomics, the study of chemical processes involving metabolites. The metabolome represents a collection of all metabolites, the end products of cellular processes, and therefore, the end results of potential disease or drug treatment; as such, metabolomics has been applied as a tool for biomarker discovery [16]. Analyzing metabolite profiles has already contributed to our understanding of depression and antidepressant treatment in mice, macaques, and humans [17-21]. Altered metabolites (e.g., decreased levels of 5-hydroxindoleacitc hydroxyindoleacetic acid (5-HIAA)) have been identified in cerebral spinal fluid [22, 23], urine, and plasma samples in depressed patients with a markedly elevated risk of suicide [24]. However, uncovering metabolites that are implicated in prophylactic efficacy has yet to be explored. Elucidating metabolic changes in the brain and plasma that are changed with prophylactic treatment may lend insight into the biological mechanisms of long-lasting resilience enhancement.

\footnotetext{
${ }^{1}$ Doctoral Program in Neurobiology and Behavior, Columbia University, New York, NY, USA; ${ }^{2}$ BERG, Framingham, MA, USA; ${ }^{3}$ Department of Psychiatry, Columbia University, New York, NY, USA and ${ }^{4}$ Division of Integrative Neuroscience, Research Foundation for Mental Hygiene, Inc. (RFMH)/New York State Psychiatric Institute (NYSPI), New York, NY, USA Correspondence: Christine A. Denny (cad2125@cumc.columbia.edu)
}

Received: 5 June 2017 Revised: 12 February 2018 Accepted: 26 February 2018

Published online: 29 March 2018 
Here, in order to begin to address the mechanism behind the efficacy of prophylactic treatment, we administered saline or ketamine $\left(30 \mathrm{mg} \mathrm{kg}^{-1}\right) 1$ week before a 3 -shock contextual fearconditioning (CFC) paradigm or before context exposure without shocks, and subsequently profiled the metabolome of both hemispheres of the prefrontal cortex (PFC) and hippocampus (HPC), as well as plasma. As we previously reported [15], prophylactic ketamine attenuated learned fear. Purine and pyrimidine metabolism was significantly altered in the HPC, PFC, and plasma following prophylactic ketamine treatment and stress as compared with saline-treated mice. Moreover, most precursors to inhibitory neurotransmitters were increased, while most precursors to excitatory neurotransmitters were decreased. Interestingly, these changes were not observed in non-stressed mice, indicating that ketamine interacts with a stressful experience to induce long-lasting changes in the metabolome and on behavior. These results point to potential metabolites as mediators in ketamine's resilience-enhancing effects, which may be easily identifiable in the clinic.

\section{MATERIALS AND METHODS}

Mice

129S6/SvEvTac mice were purchased from Taconic (Hudson, NY) at 7 weeks of age. Mice were housed 4-5 per cage in a 12-h (06:00-18:00) light-dark colony room at $22^{\circ} \mathrm{C}$. Food and water were provided ad libitum. Behavioral testing was performed during the light phase. All experiments were approved by the Institutional Animal Care and Use Committee at New York State Psychiatric Institute (NYSPI).

\section{Drugs}

A single injection of saline $(0.9 \% \mathrm{NaCl})$ or ketamine $\left(30 \mathrm{mg} \mathrm{kg}^{-1}\right)$ (Ketaset III, Ketamine $\mathrm{HCl}$ injection, Fort Dodge Animal Health, Fort Dodge, IA) was administered once at 8 weeks of age as previously described $[12,15]$. Ketamine was prepared in physiological saline and all injections were administered intraperitoneally (i.p.) in volumes of $0.1 \mathrm{cc}$ per $10 \mathrm{mg}$ body weight.

\section{Contextual fear conditioning (CFC)}

A 3-shock CFC paradigm was administered as previously described $[15,25,26]$. Mice were placed in the conditioning chamber and received 3 shocks 180,240 , and 300 s later $(2 \mathrm{~s}, 0.75 \mathrm{~mA})$ and were removed $15 \mathrm{~s}$ following the last shock. For context re-exposure, mice were placed back into the context for $180 \mathrm{~s}$. The no-shock control group underwent the same protocol, but did not receive 3 shocks during the first exposure. All sessions were scored for freezing using FreezeView2 (http://actimetrics.com/downloads/ freezeframe/). All behavioral statistical analyses are included in Table S1.

\section{Brain extraction}

Mice were sacrificed via cervical dislocation $2 \mathrm{~h}$ following CFC reexposure. Brains were extracted and frozen at $-20^{\circ} \mathrm{C}$ for $30 \mathrm{~s}$. Brains were cut using a brain matrix slicer (Cat. \# BSMAS001-1, Zivic Instruments, Pittsburgh, PA, USA). Bilateral punches were then excised from the PFC using a brain punch tissue set (Cat. \# 57401, Stoelting, Wood Dale, IL) according to the coordinates of Paxinos and Franklin (2001) in a glass dish containing ice. Left and right punches were stored separately. The HPC was manually dissected and left and right hemispheres were stored separately. Each sample was weighed. The weights of all samples are included in Table S1. All tissue samples were then stored at $-80^{\circ} \mathrm{C}$ until metabolomic analysis.

Blood plasma collection

Trunk blood was collected immediately following decapitation and spun down $(10,000 \mathrm{rpm})$ in Eppendorf tubes pre-coated with
$5 \mu \mathrm{l} 0.5 \mathrm{M}$ EDTA to obtain plasma. Samples were transferred to new tubes and stored at $-80^{\circ} \mathrm{C}$ until analysis. All tissue samples were then stored at $-80^{\circ} \mathrm{C}$ until analysis.

Preparation of brain tissue

Frozen brain tissue was transferred to a homogenization tube containing ceramic beads (Omni International, Kennesaw, GA). A volume of ice-cold $80 \% \mathrm{MeOH}$ was added to obtain a $20 \mathrm{mg} / \mathrm{ml}$ solution to each of the tubes. The tubes were snap frozen in liquid nitrogen during processing to ensure the tissue was kept cold. After a brief thawing period, the tubes were then transferred to a Bead Ruptor Homogenizer (Omni International, Kennesaw, GA). Tissue was homogenized for 2 cycles of $45 \mathrm{~s}$, with a $15 \mathrm{~s}$ rest period in between. Samples were sonicated for $10 \mathrm{~min}$ at room temperature (RT) and then centrifuged at $14,000 \mathrm{rpm}$ for $10 \mathrm{~min}$ at $4{ }^{\circ} \mathrm{C}$. All of the supernatant was removed, transferred to an Eppendorf tube, and evaporated to dryness overnight using a centrifugal evaporator. Once dry, the dried lysate was stored at $-80^{\circ} \mathrm{C}$ until analysis.

Preparation of plasma

Plasma was stored at $-80^{\circ} \mathrm{C}$ until extraction. The samples were thawed at RT for $10 \mathrm{~min}$. Thirty microliters were removed and were added to $270 \mu \mathrm{l}$ of ice-cold $80 \% \mathrm{MeOH}$. The lysate was then vortexed and spun in a centrifuge at $14,000 \mathrm{rpm}$ for $10 \mathrm{~min}$ at $4{ }^{\circ} \mathrm{C}$. One hundred microliters of supernatant were taken and transferred to a fresh Eppendorf tube and evaporated to dryness overnight using a centrifugal evaporator. Once dry, the lysate was stored at $-80^{\circ} \mathrm{C}$ until analysis.

Metabolomics analysis

For untargeted metabolomics analysis, brain samples were reconstituted in 2:1:1 acetonitrile: $\mathrm{MeOH}: \mathrm{H}_{2} \mathrm{O}$, to yield a concentration of $200 \mathrm{mg} / \mathrm{ml}$. Plasma samples were reconstituted in the same solvent, at a $30 \mu \mathrm{l}$ resuspension volume. Both were spun at $14,000 \mathrm{rpm}$ for $10 \mathrm{~min}$ at $4{ }^{\circ} \mathrm{C}$ to remove excess debris before analysis. Chromatography was performed using an Agilent 1290 Infinity UPLC. Ten microliters of each sample were injected onto a ZIC-pHILIC column (EMD Millipore, Billerica, MA) with dimensions of $150 \times 4.6 \mathrm{~mm}, 5 \mu \mathrm{m}$. Metabolites were separated using an acetonitrile/ $\mathrm{H}_{2} \mathrm{O}$ with $20 \mathrm{mM}$ ammonium carbonate $(\mathrm{pH}$ 9.2) gradient over a 29-min period. A 10-min reequilibration time was carried out in between injections. Detection was performed using an Agilent 6550 quadrupole-time-of-flight (QToF) mass spectrometer, operated in both negative and positive modes. Full scan MS data was collected from m/z 70-1000 and metabolites were identified in an untargeted manner by looking within $10 \mathrm{ppm}$ of the expected $\mathrm{m} / \mathrm{z}$ values. Real-time mass calibration was performed throughout the duration of sample analysis.

Plasma samples were also subjected to a separate analysis of metabolites involved in neurotransmission. The dried plasma samples were reconstituted in $30 \mu \mathrm{l}$ of $\mathrm{H}_{2} \mathrm{O}$. Five microliters of each sample were injected onto a Kinetix F5 column (Phenomenex, Torrance, CA) with dimensions of $100 \times 3 \mathrm{~mm}$, $2.6 \mu \mathrm{m}$. Metabolites were separated using water with $0.2 \%$ formic acid and $\mathrm{MeOH}$ gradient over a 12-min period. A reequilibration time of $3 \mathrm{~min}$ was carried out in between injections. Similarly, detection was performed using an Agilent 6550 QToF mass spectrometer, operated in both negative and positive modes (Figures S1-S3). Full scan MS data was collected and metabolites were identified using expected $\mathrm{m} /$ $z$ values, as well as confirmation against purified chemical standards. Data was processed using a publically available software package, MAVEN (https://www.ncbi.nlm.nih.gov/ pubmed/22389014). Area under the chromatographic peak for each metabolite was calculated and exported to assess for differences in metabolite abundances. 


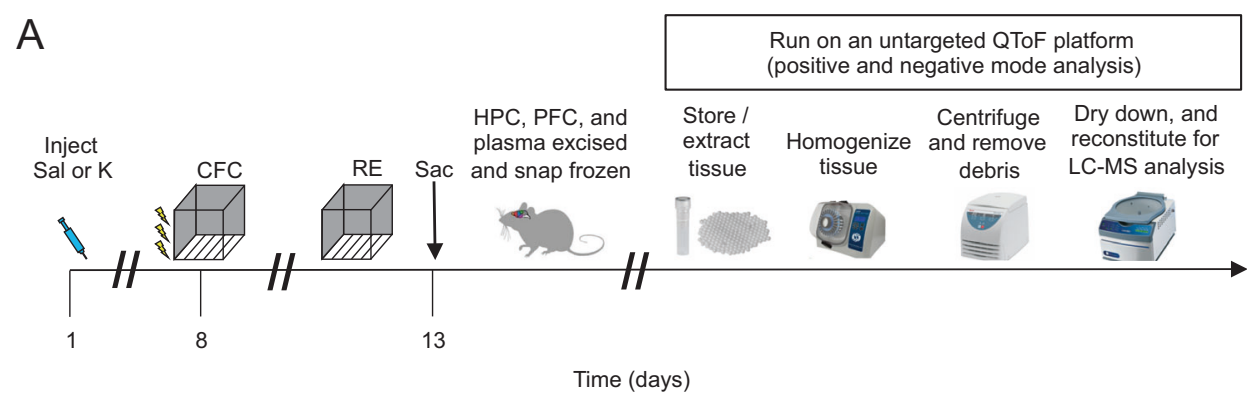

B

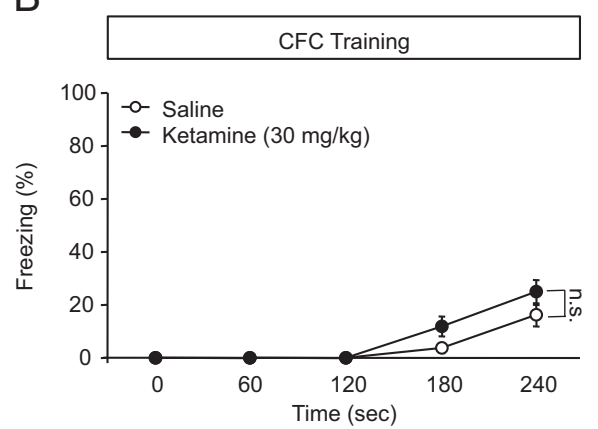

C

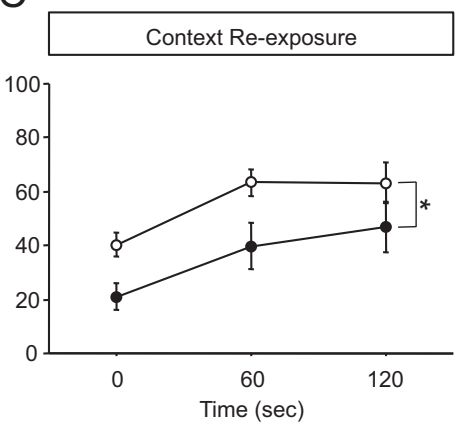

Fig. 1 Prophylactic ketamine buffers the fear response and alters a significant number of metabolites in the brain. a Experimental design. $\mathbf{b}$ Prophylactic ketamine does not alter CFC training behavior as measured by freezing. c Prophylactic ketamine administration decreases freezing behavior upon context re-exposure when compared with prophylactic saline administration. ( $n=9-10$ male mice per group). Error bars represent \pm SEM. ${ }^{*} p<0.05$. Sal saline, K ketamine, CFC contextual fear conditioning, RE re-exposure, HPC hippocampus, PFC prefrontal cortex, Sac sacrifice, QToF quadrupole-time-of-flight, LCMS liquid chromatography mass spectrometry

Pathway analysis

For each pathway analysis, the metabolites that were changed in both hemispheres or in the plasma were entered into MetaboAnalyst 3.0 using their HMDB IDs (http://www.metaboanalyst.ca/ faces/Secure/upload/PathUploadView.xhtml).

\section{Heat map}

MATLAB (https://www.mathworks.com/, Mathworks, Natick, MA) was used to transform metabolomics data for the heat maps. The function NormalizeforHeatMap takes a data input that is in the form of a matrix (size $X$ by $Y$ ) and normalizes each row to values between 0 and 1 . This function loops through each row, and at each iteration, the function first takes the minimum of each row and subtracts that from all of the values in that row. It then divides each value in that row by the maximum value of that row. The data are accumulated into a new matrix that contains all of the normalized values.

\section{Statistical analysis}

All data were analyzed using StatView 5.0 software (SAS Institute, Cary, NC) or Prism 7.0 (Graphpad Software, Inc., La Jolla, CA). Alpha was set to 0.05 for all analyses. In general, the effect of drug was analyzed using an analysis of variance (ANOVA), using repeated measures where appropriate. Unpaired $t$-tests were performed on each metabolite analyzed. All statistical tests and $p$ values for metabolomic analyses are listed in Table S1.

\section{RESULTS}

Prophylactic ketamine buffers conditioned fear responses First, to validate our previously reported effect of prophylactic ketamine on CFC, we administered a single dose of saline or ketamine $\left(30 \mathrm{mg} \mathrm{kg}^{-1}\right)$ to mice 1 week before a 3-shock CFC paradigm (Fig. 1a). Prophylactic ketamine did not alter freezing behavior during CFC encoding (Fig. 1b). As we previously reported [15], during context re-exposure, prophylactic ketamine attenuated the fear response (Fig. 1c). In another group of mice, we administered a single dose of saline or ketamine $\left(30 \mathrm{mg} \mathrm{kg}^{-1}\right)$ to mice 1 week before being exposed to the CFC context, with no shocks (Figure S4A). Prophylactic ketamine did not alter freezing behavior during context exposure or re-exposure (Figure S4B-S4C).

Prophylactic ketamine significantly alters metabolites in the PFC and HPC following stress

A number of positive and negative mode metabolites were changed in the PFC (Figure S1-S2) and HPC (Figure S3) following prophylactic ketamine administration and a CFC stressor. Notably, eight metabolites were changed in both hemispheres of the PFC and HPC (Fig. 2a, Table S2). A pathway analysis of changed metabolites in the PFC indicated that purine metabolism; phenylalanine, tyrosine, and tryptophan metabolism; and phenylalanine metabolism are most significantly changed in the PFC following prophylactic ketamine administration and stress (Fig. 2b). A pathway analysis of changed metabolites in the HPC indicated that as in the PFC, purine metabolism was also significantly altered (Fig. 2c). However, prophylactic ketamine treatment before stress also altered alanine, aspartate, and glutamate metabolism; glutamine and glutamate metabolism; and taurine and hypotaurine metabolism in the HPC (Fig. 2c).

Next, heat maps of the changed metabolites in the PFC and HPC were created for the stressed mice (Fig. 2d,e). Heat maps were used to visualize changes following ketamine treatment in the left versus right hemispheres of each brain region. A number of metabolites had altered expression following prophylactic ketamine administration when compared with prophylactic saline expression.

Prophylactic ketamine significantly alters purine metabolism following stress

Of the eight metabolites changed, six metabolites were changed in the same direction in both hemispheres of the PFC and HPC of prophylactic ketamine-treated mice that had undergone stress, 
A
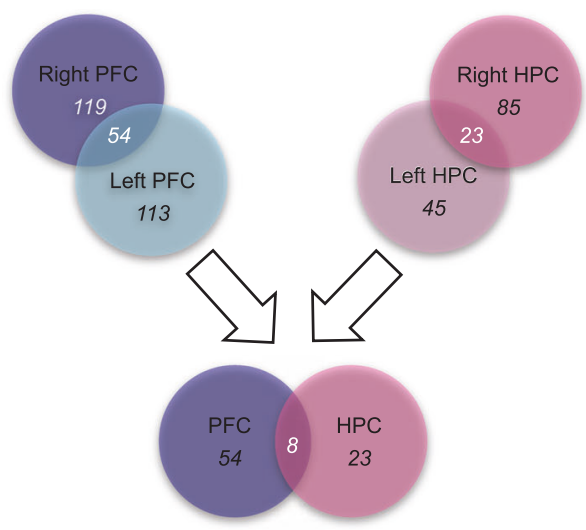

D
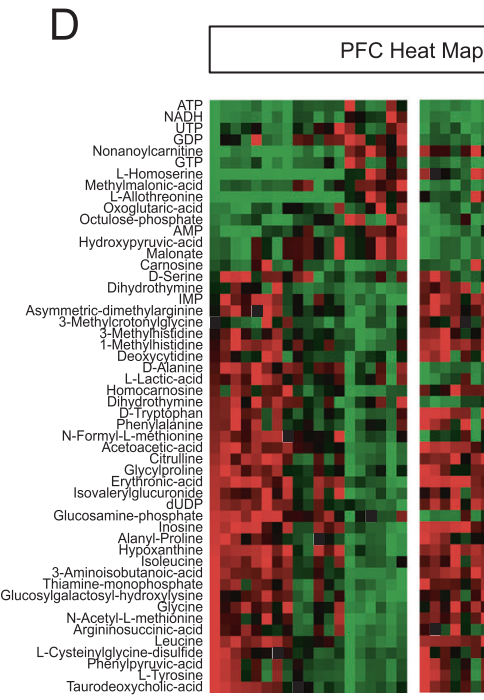

$\frac{\overline{\text { Saline }} \overline{\text { Ketamine }}}{\text { Left }}$

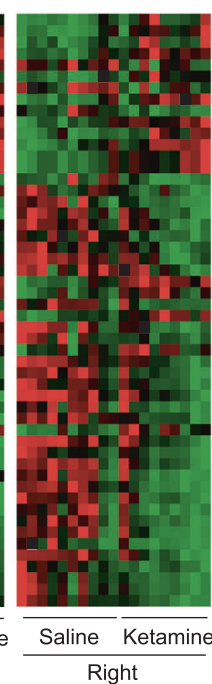

B

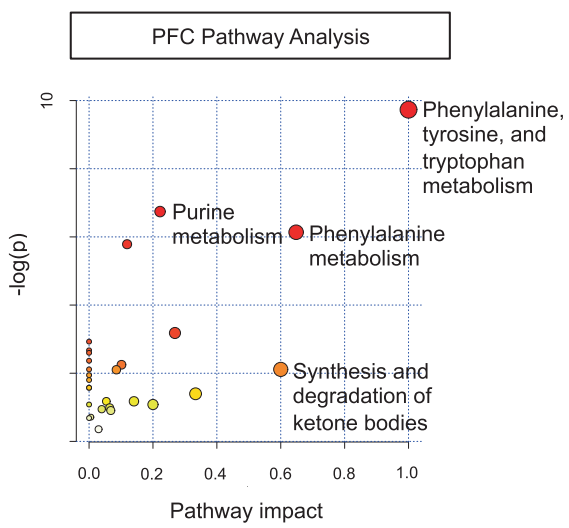

C

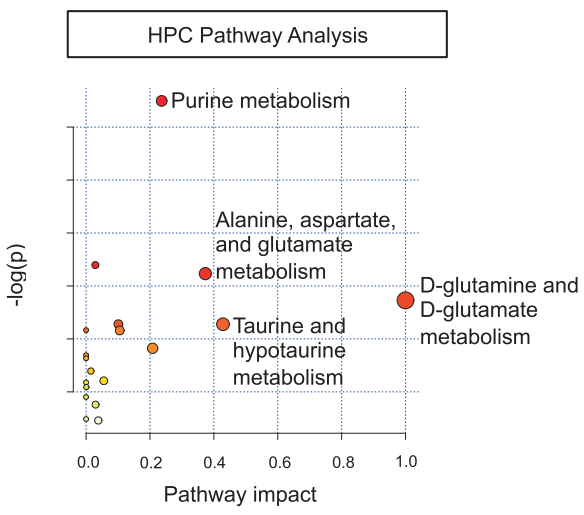

$\mathrm{E}$
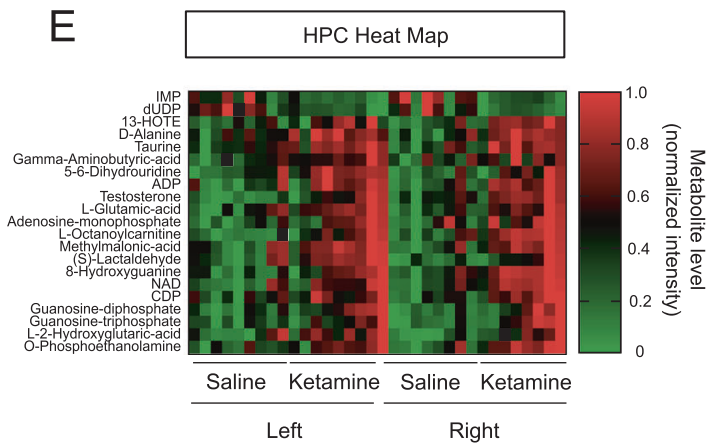

Fig. 2 Prophylactic ketamine significantly alters metabolites in the PFC and HPC following stress. a A significant number of metabolites were changed in both brain regions and in both hemispheres. A total number of eight metabolites were changed in both brain regions and in both hemispheres. b A pathway analysis of changed metabolites in the PFC. Purine metabolism; phenylalanine, tyrosine, and tryptophan metabolism; and phenylalanine metabolism are most significantly changed in the PFC following prophylactic ketamine administration. c A pathway analysis of changed metabolites in the HPC. Purine metabolism; alanine, aspartate, and glutamate metabolism; and glutamine and glutamate metabolism are most significantly changed in the HPC following prophylactic ketamine administration. d A heat map of metabolites changed in the PFC. e A heat map of metabolites changed in the HPC. ( $n=9-10$ male mice per group). HPC hippocampus, PFC prefrontal cortex, ATP adenosine triphosphate, NADH nicotinamide adenine dinucleotide, UTP uridine triphosphate, GDP guanosine diphosphate, GTP guanosine triphosphate, AMP adenosine monophosphate, IMP inosine monophosphate, dUDP deoxyuridine-diphosphate, 13-HOTE 13-OH-9Z,11E,15Z-octadecatrienoic acid, NAD nicotinamide adenine dinucleotide, CDP cytidine diphosphate, ADP adenosine diphosphate

and these metabolites were involved in purine and pyrimidine biosynthesis (Table S2). We first analyzed individual purine metabolites in the PFC and HPC. Purine precursors and nucleotides were significantly altered in both hemispheres of the PFC (Fig. 3a-g) and HPC (Fig. 3h-l). Specifically, inosine metabolites were decreased following prophylactic ketamine administration, and the downstream metabolites adenosine and guanosine metabolites were increased. However, these changes were not observed in a group of mice that received prophylactic ketamine without stress (Figure S4).
Prophylactic ketamine significantly alters pyrimidine metabolism following stress

Pyrimidine metabolites were next analyzed in both the PFC (Fig. 4a-e) and HPC (Fig. 4f-h). Since both the PFC and HPC showed alterations in deoxyuridine diphosphate (dUDP), we performed a regression analysis on dUDP expression and freezing behavior in both the PFC (Fig. 4i) and in the HPC (Fig. 4j). In the PFC, dUDP expression is positively correlated with freezing levels upon context re-exposure in mice administered prophylactic ketamine prior to stress. These alterations in pyrimidine 

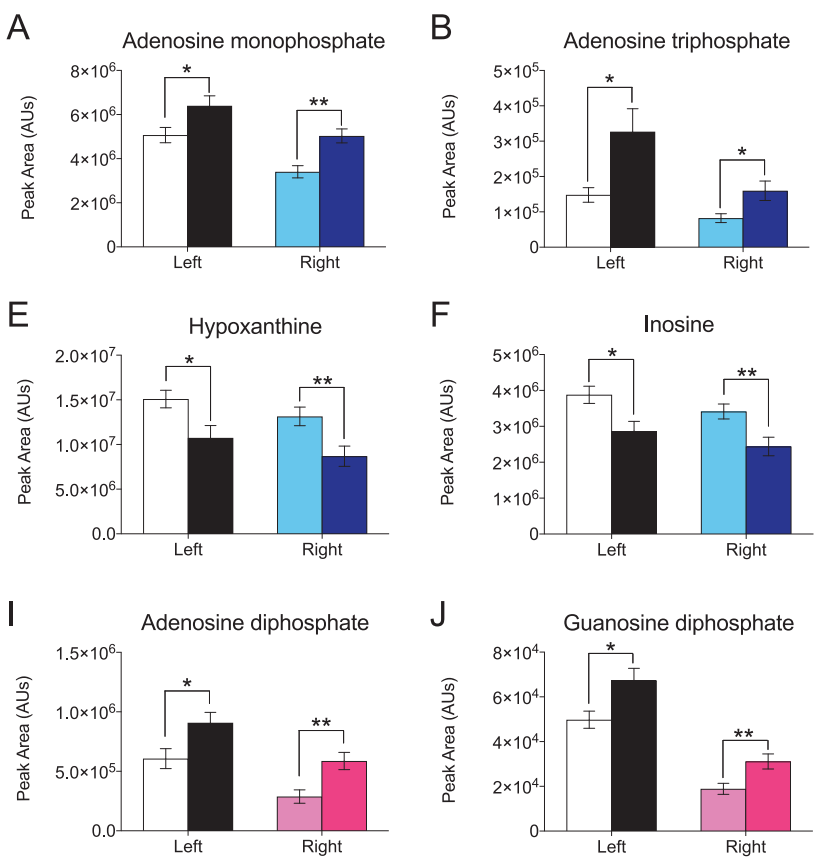

PFC: $\square$ Sal, Left

$\mathrm{K}(30 \mathrm{mg} / \mathrm{kg})$, Left

$\square$ Sal, Right $\square$ K(30 mg/kg), Right
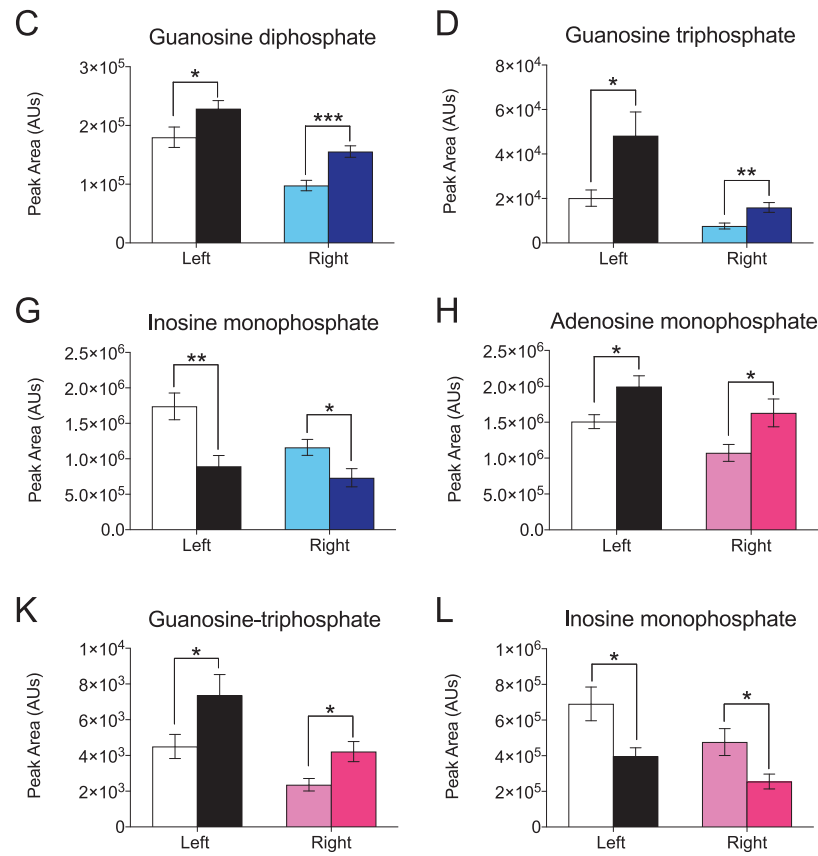

HPC: $\square$ Sal, Left

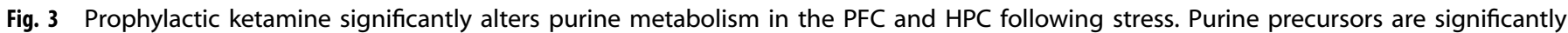
decreased and nucleotides are significantly increased in both hemispheres of the a-g PFC and h-I HPC following prophylactic ketamine administration. ( $n=9-10$ male mice per group). Error bars represent \pm SEM. ${ }^{*} p<0.05,{ }^{* *} p<0.01$, ${ }^{* *} p<0.001$. Sal saline, K ketamine

metabolism were not observed in mice that received prophylactic ketamine without stress (Figure S4).

Prophylactic ketamine significantly alters purine and pyrimidine metabolism in plasma after stress

We next predicted that the effects of prophylactic ketamine might be apparent in the periphery as well as in the brain. Therefore, metabolomics analysis was next performed on plasma samples of stressed and non-stressed mice. A pathway analysis was first performed of changed metabolites indicating that purine metabolism, pyrimidine metabolism, the citrate cycle (TCA cycle), and the pentose phosphate pathway were most significantly changed following prophylactic ketamine administration and stress (Fig. 5a). A heat map was created of all metabolites changed in the plasma between the two groups (Fig. 5b). Prophylactic ketamine administration significantly increased purine metabolites in the plasma (Fig. $5 \mathrm{c}-\mathrm{l}$ ). Moreover, with the exception of 5,6-dihydrouridine, prophylactic ketamine administration increased expression of pyrimidine metabolites in the plasma (Fig. $5 \mathrm{~m}-\mathrm{q}$ ). Interestingly, when the mice were not administered shocks, no changes in purines or pyrimidine metabolites were observed, suggesting that prophylactic ketamine interacts with stress to produce long-term peripheral changes in purine and pyrimidine metabolism (Figure S4).

Amino acid-derived neurotransmitters and precursors are significantly changed following prophylactic ketamine and stress in the PFC and HPC

All amino-acid-derived neurotransmitters and their precursors were next analyzed for alterations following prophylactic ketamine administration (Table S3). Interestingly, following stress, almost all inhibitory neurotransmitter metabolites were increased (e.g., alanine, gamma-aminobutyric acid (GABA), taurine), and nearly all excitatory neurotransmitter metabolites were decreased (e.g., serine, tyrosine, and phenylalanine). The main exception to this observation was glutamic acid, a precursor to GABA.
Therefore, it is possible that the increase in glutamic acid is directly related to the increase in GABA. In contrast, in nonstressed mice, only the inhibitory neurotransmitter metabolite taurine was changed. These data suggest that prophylactic ketamine and stress may increase inhibitory tone in the brain following administration, resulting in long-lasting protection.

Amino acid-derived neurotransmitters and precursors are significantly changed following prophylactic ketamine and stress in plasma

As performed on brain tissue, amino-acid-derived neurotransmitters and their precursors in plasma were next analyzed for alterations following prophylactic ketamine administration (Table S3). A number of amino-acid-derived neurotransmitters and precursors were altered, but only in stressed mice. Interestingly, as in the brain tissue, two excitatory neurotransmitters serine and glutamic acid were decreased. However, unlike in the brain tissue, GABA, an inhibitory neurotransmitter, was decreased. These data suggest that metabolite profiling of plasma samples may indicate effectiveness of prophylactic treatment.

\section{DISCUSSION}

Here, we have shown that a single injection of ketamine before a 3-shock CFC protocol attenuated learned fear and produced longlasting changes in the metabolite profile of PFC and HPC tissues, as well as of plasma. Interestingly, a single injection of ketamine without stress did not produce similar long-lasting changes in the PFC, HPC, or plasma, suggesting that prophylactic ketamine may interact with stress to induce long-lasting metabolic changes, especially in the periphery, that affect behavior. To our knowledge, this is the first study that identifies metabolite alterations and biomarker candidates for prophylactic ketamine efficacy in mice.

Purine and pyrimidine metabolism was affected not only in the PFC and in the HPC, but also in plasma of prophylactic ketaminetreated stressed mice. Genetically determined aberrations in 
PFC: $\square$ Sal, Left

A

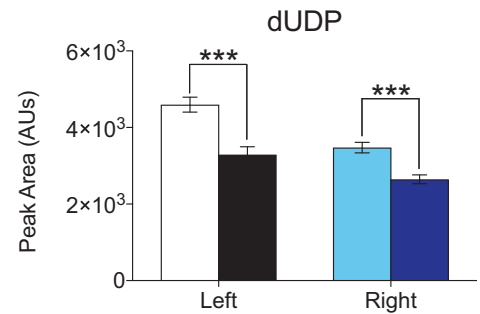

B

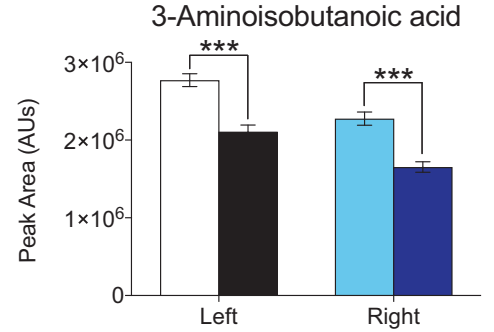

C

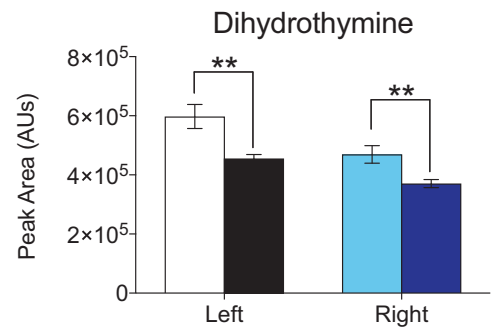

D

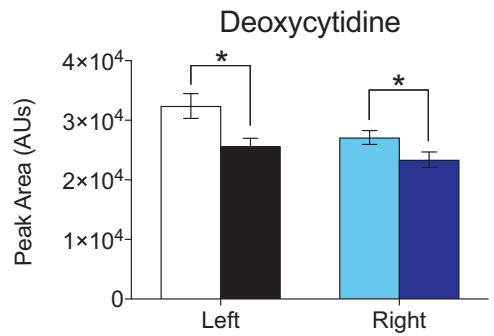

E

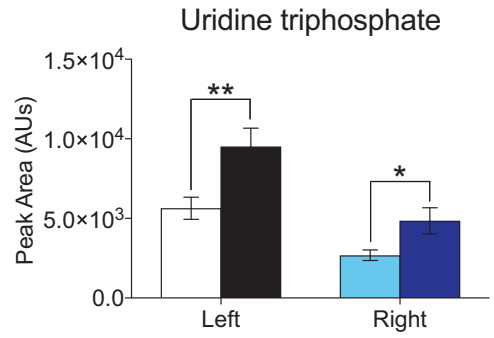

F dUDP

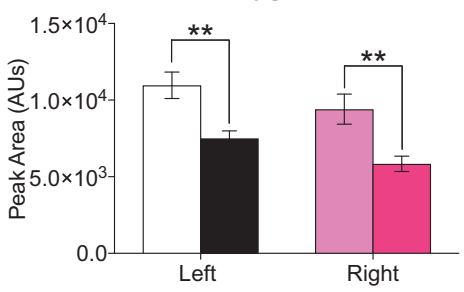

G

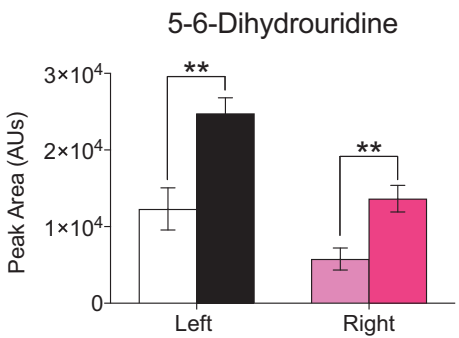

$\mathrm{H}$
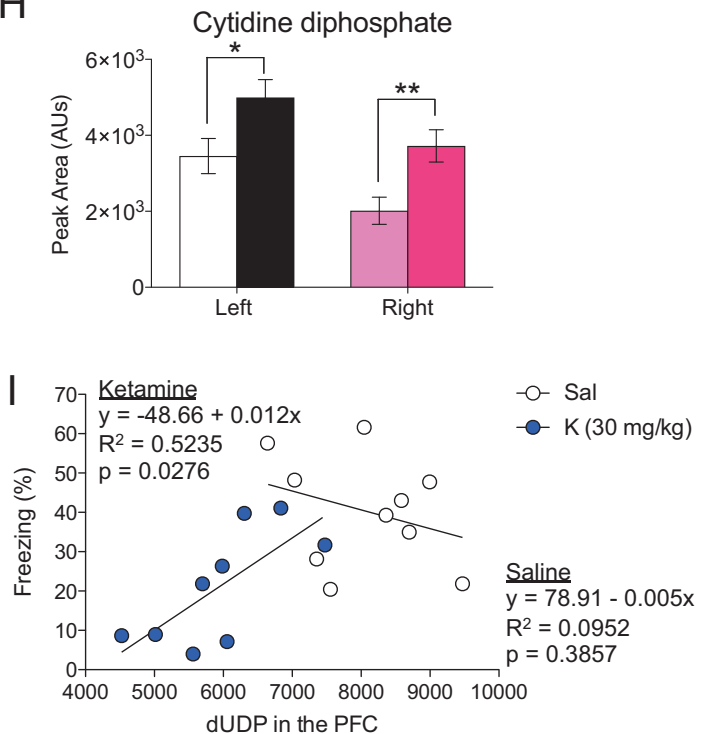

$J$

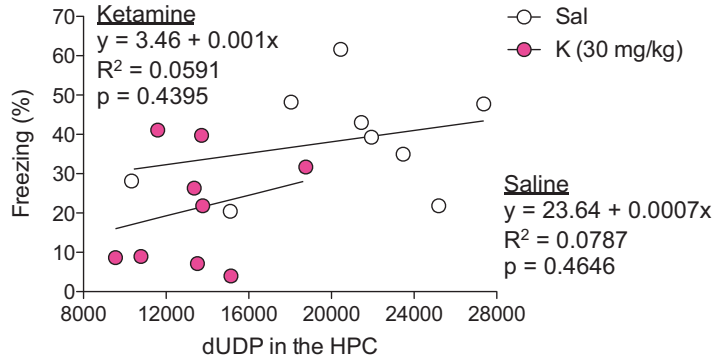

Fig. 4 Prophylactic ketamine significantly alters pyrimidine metabolism in the PFC and HPC following stress. a-e Pyrimidine metabolites are significantly altered in both hemispheres of the PFC following prophylactic ketamine administration. $\mathbf{f}-\mathbf{h}$ Pyrimidine metabolites are significantly altered in both hemispheres of the HPC following prophylactic ketamine administration. $\mathbf{i}$, $\mathbf{j}$ The amount of dUDP in both hemispheres of the PFC, but not the HPC, is positively correlated with freezing levels upon context re-exposure in CFC in mice administered prophylactic ketamine and stress. ( $n=9-10$ male mice per group). Error bars represent \pm SEM. ${ }^{*} p<0.05,{ }^{* *} p<0.01,{ }^{* * *} p<0.001$. Sal saline, K ketamine, PFC prefrontal cortex, HPC hippocampus, dUDP deoxyuridine-diphosphate 

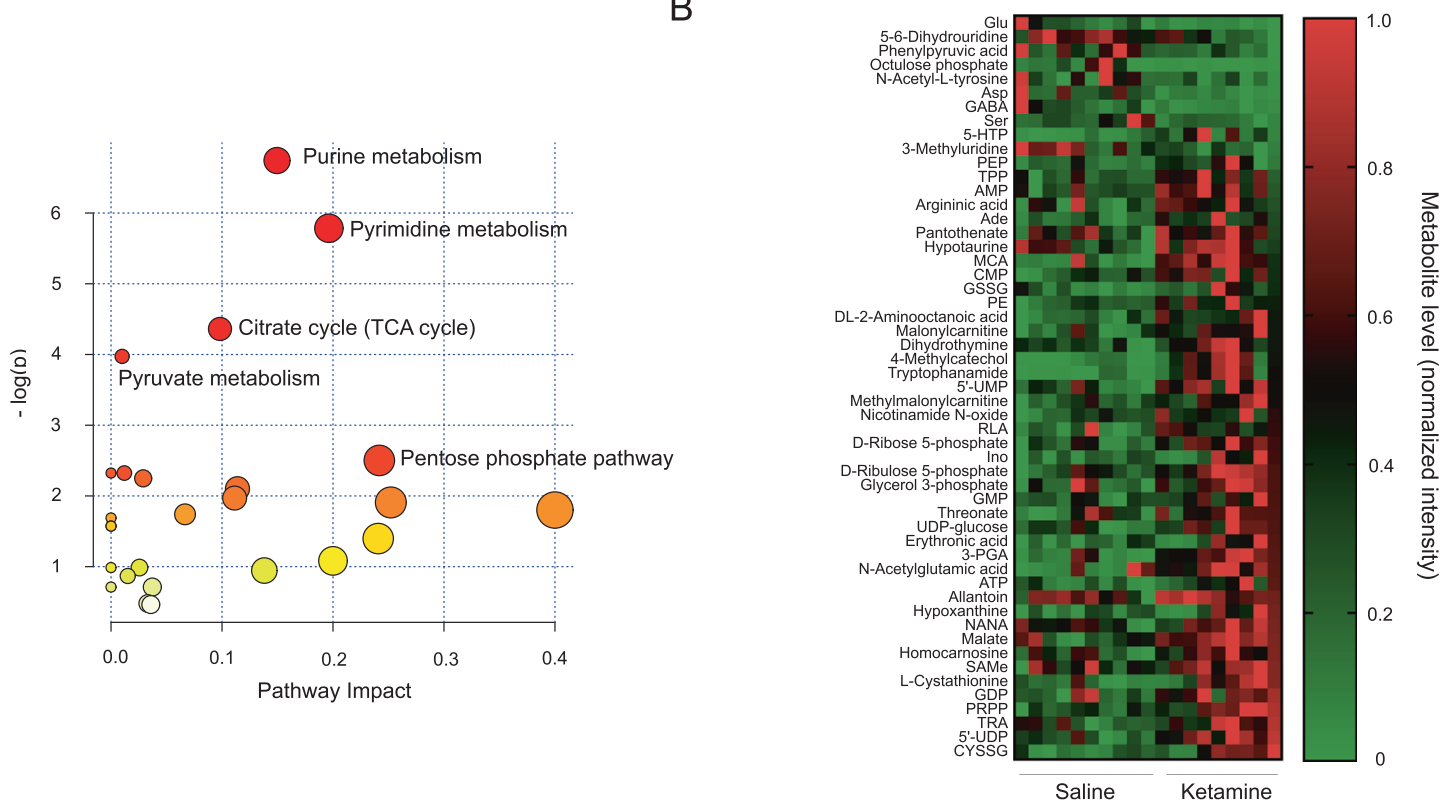
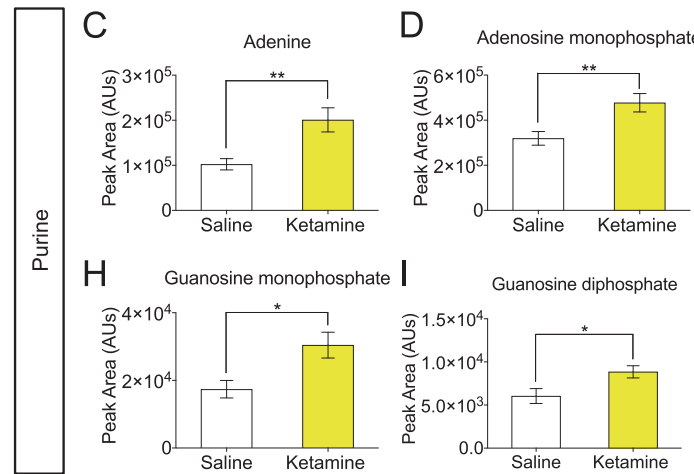

E
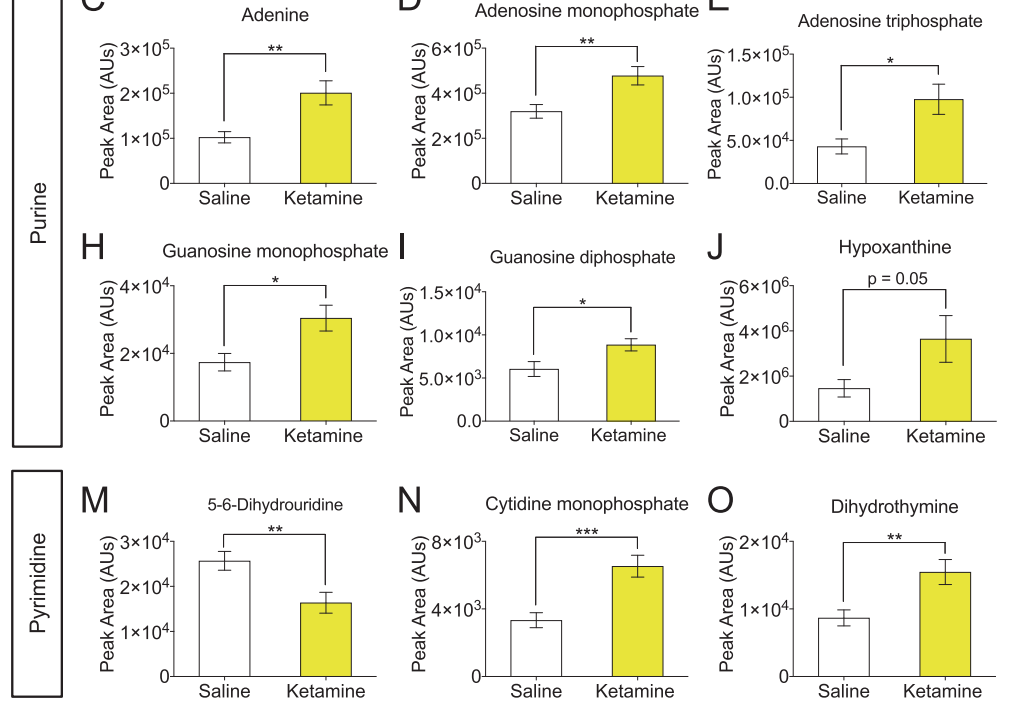

$\mathrm{F}$
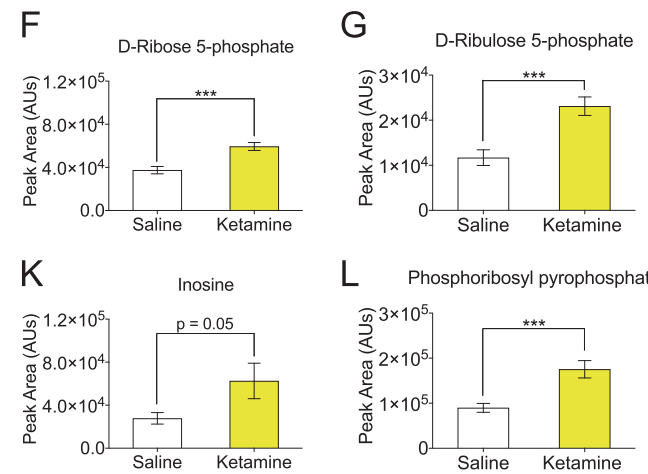

L Phosphoribosyl pyrophosphate
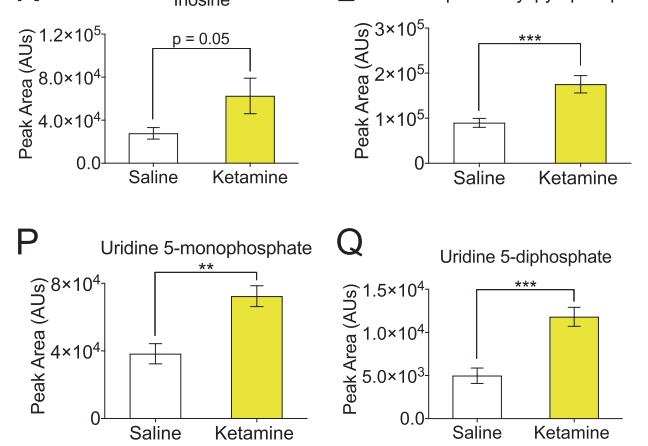

Fig. 5 Prophylactic ketamine significantly alters purine and pyrimidine metabolism in plasma following stress. a A pathway analysis of changed metabolites in the plasma. Purine metabolism; pyrimidine metabolism; and the TCA cycle were most significantly changed following prophylactic ketamine administration. b A heat map of changed metabolites in the plasma. c-I Purine metabolites significantly increased in the plasma following prophylactic ketamine administration. $\mathbf{m}-\mathbf{q}$ Pyrimidine metabolites significantly increased in the plasma following prophylactic ketamine administration. ( $n=9-10$ male mice per group). Error bars represent \pm SEM. ${ }^{*} p<0.05,{ }^{* *} p<0.01,{ }^{* * *} p<0.001$. TCA tricarboxylic acid, Glu glutamate, Asp aspartate, GABA gamma-aminobutyric acid, Ser serine, 5-HTP 5-hydroxytryptophan, PEP phosphoenolpyruvic acid, TPP thiamine pyrophosphate, AMP adenosine monophosphate, Ade adenosine, MCA monochloroacetic acid, CMP cytidine monophosphate, GSSG glutathione disulfide, PE phosphatidylethanolamine, 5'-UMP 5'-uridine monophosphate; RLA, R(+)-enantiomer lipoic acid, Ino inosine, GMP guanosine monophosphate, 3-PGA 3-phosphoglyceric acid, ATP adenosine triphosphate, NANA $\mathrm{N}$-acetylneuraminic acid, SAMe S-adenosyl methionine, GDP guanosine diphosphate, PRPP 5-phospho-alpha-D-ribosyl 1-pyrophosphate, TRA tiaramide, 5'-UDP 5'-uridine diphosphate, CYSSG cysteine-glutatione disulfide

purine and pyrimidine metabolism have been associated with neurological dysfunction [27-29] and psychiatric disorders [30, 31]. Purine and pyrimidine metabolism has also recently been implicated in antidepressant treatment response in mice and humans [18]. In this study, a commonly used selective serotonin reuptake inhibitor (SSRI), paroxetine, was administered to DBA/2J mice for 28 days. Drug non-responder and responder mice were identified, and the metabolomic profile was assessed in the HPC and plasma. Their results suggest that chronic SSRI treatment differentially affects purine and pyrimidine metabolism, which may explain the heterogeneous antidepressant treatment response, and represents a potential biosignature of the disease. Specifically, the authors observed decreased purine and pyrimidine metabolism in antidepressant non-responders, and increased metabolism in responders. Here, we have similarly assessed efficacy of the rapid-acting antidepressant ketamine, but when administered prior to stress. The effect of prophylactic ketamine on purine and pyrimidine metabolism after stress suggests that several mechanisms of successful antidepressant treatment may overlap with prophylactic responsiveness. Additionally, to further 
assess how changes in the metabolome relate to behavioral output, we correlated the freezing levels of stressed mice with the amount of the pyrimidine dUDP in both hemispheres of the PFC and HPC. We found that dUDP levels positively correlated with freezing levels upon context re-exposure, but only in the PFC of mice administered ketamine, suggesting that the alterations in the metabolome induced by ketamine and stress may directly influence pathological states.

Prophylactic ketamine increased precursors to inhibitory neurotransmitters, while decreasing most precursors to excitatory neurotransmitters in stressed mice. Increasing evidence demonstrates that MDD is associated with an imbalance of excitatory and inhibitory neurotransmitters glutamate and GABA [32]. Specifically, glutamic acid and GABA dysregulation is a hallmark of MDD pathology [33, 34], as well as of PTSD and other panic and social anxiety disorders $[35,36]$. Interestingly, in a model of chronic social defeat stress (CSDS), mice that exhibited depressive-like behavior showed a reduction in glutamate in the GABAergic pathway, suggesting that glutamatergic disorders may be implicated in susceptibility to stress [37]. Collectively, these data suggest that prophylactic ketamine may be increasing stress resilience by regulating GABA and glutamate neurotransmission and therefore, preventing stress-induced phenotypes.

No previous studies have identified the alterations in the metabolome that occur upon administering a resilienceenhancing pharmaceutical, as the current study sought to assess. However, previous studies have been conducted in regards to ketamine's rapid-acting antidepressant effects and the resultant metabolomic and proteomic changes. Specifically, adenosine has been shown to mediate antidepressant action of ketamine, and that enhancement of the adenosine $A_{1}$ receptors $\left(A_{1} R s\right)$ in forebrain neurons of mice produced resilience-enhancing effects [38]. In general, adenosine has been known to act as an antiinflammatory agent involved in neuroprotection, and it is hypothesized that imbalanced levels of adenosine-5'-triphosphate (ATP) and adenosine in the extracellular space may lead to neuropsychiatric diseases [31]. These data are in agreement with our results demonstrating that prophylactic ketamine administration increases levels of adenosine monophosphate (AMP) and ATP in both hemispheres of the PFC and HPC, and in the plasma.

Thyrotropin-releasing hormone (TRH) and TRH-like peptides, which are potential therapeutic targets for MDD, are also increased in various brain regions, including the PFC and HPC, upon ketamine administration [39]. It has also been demonstrated that in as rapidly as $2 \mathrm{~h}$ post-ketamine injection, hippocampal pathways including glycolysis, pentose phosphate pathway, and citrate cycle are impacted [20]. Importantly, the pentose phosphate pathway is known to be important for purine formation. Here, we show that ribose and ribulose phosphate, pentose phosphate pathway intermediates, are significantly changed in plasma, which is in agreement with Weckmann et al.'s study demonstrating the role of ketamine on the pentose phosphate pathway and purine formation. Thus, there may be a cascade of changes occurring after ketamine administration that are activated during stress, which could explain its long-lasting resilience-enhancing effects, such as changes in the pentose phosphate pathway that lead to changes in purine formation.

The use of metabolite supplements in the treatment of depression has been attempted, as metabolic syndromes have been associated with MDD [40, 41] and PTSD [42]. Interestingly, one clinical study found that up to two-thirds of TRD patients may demonstrate at least one metabolic abnormality [24]. Systemic folate deficiency has been of particular relevance to this study, as cerebral folate deficiency (CFD) was the most common in the TRD patients. Typically, CFD syndromes present as developmental and psychiatric disorders [38, 43]. Folate, a water-soluble B-vitamin, is involved in nearly 100 metabolic reactions including the purine synthetic pathway [44], and is necessary for the biosynthesis of monoamine neurotransmitters serotonin, epinephrine, and dopamine [38]. Low folate levels have been associated with reduced response to antidepressants, and have been correlated with MDD symptomology; thus, folate supplements such as folic acid or methylfolate have been prescribed to MDD patients [45]. The influence of folate on monoamine neurotransmitters is reflected in our data, as folate changes may have influenced changes we observed in neurotransmitters such as tyrosine and phenylalanine in the brain, which are precursors to dopamine, norepinephrine, and epinephrine.

The identification of specific metabolite markers associated with psychiatric disease using metabolomics platforms has only been recently pursued. There are an emerging series of studies assessing urinary and peripheral blood metabolite biomarkers to aid in diagnosis of MDD [46-50]. Prior to metabolomics studies, research focused on identifying genomic markers of MDD, but efforts have not yet yielded diagnostic and treatment biomarkers in the genome [51]. Interestingly, we found that prophylactic ketamine significantly altered metabolites in both the brain and the plasma after stress. Importantly, prophylactic ketamine alone does not induce the same changes in metabolites, but rather, these changes are triggered only after the experience of a stressor. This is noteworthy, as it suggests that there are peripheral changes with prophylactic treatment and stress that may be identified in the clinic.

Overall, the present study may lead to novel insights on how ketamine treatment prior to a stressor may alter metabolic pathways that are involved in neurotransmission, and how these pathways are implicated in mood disorders. These data suggest that a single dose of ketamine induces a cascade of effects following a stressor that may underlie its long-lasting resilience against stress-induced disorders. These results may elucidate potential biological processes in the brain and plasma that are critical for maintaining resilience against stressors.

\section{ACKNOWLEDGEMENTS}

We thank members of the laboratory for insightful comments on this project and manuscript. JCM was supported by the Neurobiology \& Behavior Research Training Grant T32 HD007430-19 and by a DGE 16-44869. CTL and AM were supported by an NIH DP5 OD017908. CAD was supported by an NIH DP5 OD017908, a National Alliance for Research on Schizophrenia and Depression (NARSAD) Young Investigator Grant from the Brain \& Behavior Research Foundation (BBRF) (P\&S Investigator), New York Stem Cell Science (NYSTEM C-029157), and a gift from For the Love of Travis, Inc.

\section{ADDITIONAL INFORMATION}

The online version of this article (https://doi.org/10.1038/s41386-018-0043-7) contains supplementary material, which is available to authorized users.

Conflict of interest: $A M$ and CTL reported no biomedical financial interests or potential conflicts of interests. $\mathrm{CH}, \mathrm{AK}, \mathrm{NRN}$, and MAK are employees of BERG LLC and own stock. NRN is a founder of BERG. JCM, RAB, and CAD are named on nonprovisional patent applications for the prophylactic use of ketamine against stressrelated psychiatric disorders.

\section{REFERENCES}

1. Berman RM, Cappiello A, Anand A, Oren DA, Heninger GR, Charney DS, et al. Antidepressant effects of ketamine in depressed patients. Biol Psychiatry. 2000;47:351-4.

2. Price RB, Nock MK, Charney DS, Mathew SJ. Effects of intravenous ketamine on explicit and implicit measures of suicidality in treatment-resistant depression. Biol Psychiatry. 2009;66:522-6.

3. Zarate CA Jr., Singh JB, Carlson PJ, Brutsche NE, Ameli R, Luckenbaugh DA, et al. A randomized trial of an $\mathrm{N}$-methyl-D-aspartate antagonist in treatment-resistant major depression. Arch General Psychiatry. 2006;63:856-64.

4. Feder A, Parides MK, Murrough JW, Perez AM, Morgan JE, Saxena S, et al. Efficacy of intravenous ketamine for treatment of chronic posttraumatic stress disorder: a randomized clinical trial. JAMA Psychiatry. 2014;71:681-8. 
5. Murrough JW, Soleimani L, DeWilde KE, Collins KA, Lapidus KA, lacoviello BM, et al. Ketamine for rapid reduction of suicidal ideation: a randomized controlled trial. Psychol Med. 2015;45:3571-80.

6. Diazgranados $\mathrm{N}$, Ibrahim $\mathrm{L}$, Brutsche NE, Newberg A, Kronstein P, Khalife $\mathrm{S}$, et al. A randomized add-on trial of an N-methyl-D-aspartate antagonist in treatmentresistant bipolar depression. Arch General Psychiatry. 2010;67:793-802.

7. Zarate CA Jr., Brutsche NE, Ibrahim L, Franco-Chaves J, Diazgranados N, Cravchik A, et al. Replication of ketamine's antidepressant efficacy in bipolar depression: a randomized controlled add-on trial. Biol Psychiatry. 2012;71:939-46.

8. Ionescu DF, Luckenbaugh DA, Niciu MJ, Richards EM, Zarate CA Jr.. A single infusion of ketamine improves depression scores in patients with anxious bipolar depression. Bipolar Disord. 2015;17:438-43.

9. Howlett JR, Stein MB. Prevention of trauma and stressor-related disorders: a review. Neuropsychopharmacology. 2016;41:357-69.

10. Knoll AT, Meloni EG, Thomas JB, Carroll FI, Carlezon WA Jr.. Anxiolytic-like effects of kappa-opioid receptor antagonists in models of unlearned and learned fear in rats. J Pharmacol Exp Ther. 2007;323:838-45.

11. Van't Veer A, Carlezon WA Jr. Role of kappa-opioid receptors in stress and anxiety-related behavior. Psychopharmacology. 2013;229:435-52.

12. Brachman RA, McGowan JC, Perusini JN, Lim SC, Pham TH, Faye C, et al. Ketamine as a prophylactic against stress-induced depressive-like behavior. Biol Psychiatry. 2016;79:776-86

13. Amat J, Dolzani SD, Tilden S, Christianson JP, Kubala KH, Bartholomay K, et al. Previous ketamine produces an enduring blockade of neurochemical and behavioral effects of uncontrollable stress. J Neurosci: Off J Soc Neurosci. 2016:36:153-61.

14. Soumier A, Carter RM, Schoenfeld TJ, Cameron HA (2016). New hippocampal neurons mature rapidly in response to ketamine but are not required for its acute antidepressant effects on neophagia in rats. eNeuro 3. 2016 Mar 31;3. pii: ENEURO.0116-15.2016

15. McGowan JC, LaGamma CT, Lim SC, Tsitsiklis M, Neria Y, Brachman RA, et al. Prophylactic ketamine attenuates learned fear. Neuropsychopharmacology. 2017:42:1577-89.

16. Johnson $\mathrm{CH}$, Ivanisevic J, Siuzdak G. Metabolomics: beyond biomarkers and towards mechanisms. Nat Rev Mol Cell Biol. 2016;17:451-9.

17. Pan X, Zeng X, Hong J, Yuan C, Cui L, Ma J, et al. Effects of ketamine on metabolomics of serum and urine in cynomolgus macaques (Macaca fascicularis). J Am Assoc Lab Anim Sci. 2016;55:558-64.

18. Park DI, Dournes C, Sillaber I, Uhr M, Asara JM, Gassen NC, et al. Purine and pyrimidine metabolism: convergent evidence on chronic antidepressant treatment response in mice and humans. Sci Rep. 2016;6:35317.

19. Rotroff DM, Corum DG, Motsinger-Reif A, Fiehn O, Bottrel N, Drevets WC, et al. Metabolomic signatures of drug response phenotypes for ketamine and esketamine in subjects with refractory major depressive disorder: new mechanistic insights for rapid acting antidepressants. Transl Psychiatry. 2016;6:e894.

20. Weckmann K, Labermaier C, Asara JM, Muller MB, Turck CW. Time-dependent metabolomic profiling of ketamine drug action reveals hippocampal pathway alterations and biomarker candidates. Transl Psychiatry. 2014;4:e481.

21. Wen $C$, Zhang M, Zhang $Y$, Sun F, Ma J, Hu L, et al. Brain metabolomics in rats after administration of ketamine. Biomed Chromatogr: BMC. 2016;30:81-84.

22. Asberg M, Traskman L, Thoren P. 5-HIAA in the cerebrospinal fluid. A biochemical suicide predictor? Arch General Psychiatry. 1976;33:1193-7.

23. Pan L, McKain BW, Madan-Khetarpal S, McGuire M, Diler RS, Perel JM, et al. (2011). GTP-cyclohydrolase deficiency responsive to sapropterin and 5-HTP supplementation: relief of treatment-refractory depression and suicidal behaviour. $B M J$ Case Rep. 2011 Jun 9;2011. pii: bcr0320113927. https://doi.org/10.1136/ bcr.03.2011.3927.

24. Pan LA, Martin P, Zimmer T, Segreti AM, Kassiff S, McKain BW, et al. Neurometabolic disorders: potentially treatable abnormalities in patients with treatmentrefractory depression and suicidal behavior. Am J Psychiatry. 2017;174:42-50.

25. Denny CA, Kheirbek MA, Alba EL, Tanaka KF, Brachman RA, Laughman KB, et al. Hippocampal memory traces are differentially modulated by experience, time, and adult neurogenesis. Neuron. 2014;83:189-201.

26. Drew MR, Denny CA, Hen R. Arrest of adult hippocampal neurogenesis in mice impairs single- but not multiple-trial contextual fear conditioning. Behav Neurosci. 2010;124:446-54.

27. Jinnah HA, Sabina RL, Van Den Berghe G. Metabolic disorders of purine metabolism affecting the nervous system. Handb Clin Neurol. 2013;113:1827-36.
28. Kelley RE, Andersson HC. Disorders of purines and pyrimidines. Handb Clin Neurol. 2014;120:827-38.

29. Micheli V, Camici M, Tozzi MG, Ipata PL, Sestini S, Bertelli M, et al. Neurological disorders of purine and pyrimidine metabolism. Curr Top Med Chem. 2011;11:923-47.

30. Astakhova LN, Asanova NK. [Borderline neuropsychiatric disorders in purine metabolism disorders in children]. Zh Nevropatol i psikhiatrii Im SS Korsakova (Mosc, Russ: 1952). 1985;85:421-6.

31. Cieslak M, Czarnecka J, Roszek K. The roles of purinergic signaling in psychiatric disorders. Acta Biochim Pol. 2016;63:1-9.

32. Hasler G, van der Veen JW, Tumonis T, Meyers N, Shen J, Drevets WC. Reduced prefrontal glutamate/glutamine and gamma-aminobutyric acid levels in major depression determined using proton magnetic resonance spectroscopy. Arch General Psychiatry. 2007;64:193-200.

33. Abdallah CG, Jiang L, De Feyter HM, Fasula M, Krystal JH, Rothman DL, et al. Glutamate metabolism in major depressive disorder. Am J Psychiatry. 2014;171: 1320-7.

34. Pehrson AL, Sanchez C. Altered gamma-aminobutyric acid neurotransmission in major depressive disorder: a critical review of the supporting evidence and the influence of serotonergic antidepressants. Drug Des, Dev Ther. 2015;9:603-24.

35. Meyerhoff DJ, Mon A, Metzler T, Neylan TC. Cortical gamma-aminobutyric acid and glutamate in posttraumatic stress disorder and their relationships to selfreported sleep quality. Sleep. 2014;37:893-900.

36. Averill LA, Purohit P, Averill CL, Boesl MA, Krystal JH, Abdallah CG. Glutamate dysregulation and glutamatergic therapeutics for PTSD: evidence from human studies. Neurosci Lett. 2016;649:147-55.

37. Wang W, Guo H, Zhang SX, Li J, Cheng K, Bai SJ, et al. Targeted metabolomic pathway analysis and validation revealed glutamatergic disorder in the prefrontal cortex among the chronic social defeat stress mice model of depression. J Proteome Res. 2016;15:3784-92.

38. Serchov T, Clement HW, Schwarz MK, lasevoli F, Tosh DK, Idzko M, et al. Increased signaling via adenosine $\mathrm{A} 1$ receptors, sleep deprivation, imipramine, and ketamine inhibit depressive-like behavior via induction of homer1a. Neuron. 2015;87:549-62.

39. Pekary AE, Sattin A, Lloyd RL. Ketamine modulates TRH and TRH-like peptide turnover in brain and peripheral tissues of male rats. Peptides. 2015;69: $66-76$.

40. Marazziti D, Rutigliano G, Baroni S, Landi P, Dell'Osso L. Metabolic syndrome and major depression. CNS Spectr. 2014;19:293-304.

41. Martinac M, Pehar D, Karlovic D, Babic D, Marcinko D, Jakovljevic M. Metabolic syndrome, activity of the hypothalamic-pituitary-adrenal axis and inflammatory mediators in depressive disorder. Acta Clin Croat. 2014;53:55-71.

42. Bartoli F, Carra G, Crocamo C, Carretta D, Clerici M. Metabolic syndrome in people suffering from posttraumatic stress disorder: a systematic review and metaanalysis. Metab Syndr Relat Disord. 2013;11:301-8.

43. Ramaekers V, Sequeira JM, Quadros EV. Clinical recognition and aspects of the cerebral folate deficiency syndromes. Clin Chem Lab Med. 2013;51:497-511.

44. Pan L, Vockley J. Neuropsychiatric symptoms in inborn errors of metabolism: incorporation of genomic and metabolomic analysis into therapeutics and prevention. Curr Genet Med Rep. 2013;1:65-70.

45. Nelson JC. The evolving story of folate in depression and the therapeutic potential of I-methylfolate. Am J Psychiatry. 2012;169:1223-5.

46. Lee MY, Kim EY, Kim SH, Cho KC, Ha K, Kim KP, et al. Discovery of serum protein biomarkers in drug-free patients with major depressive disorder. Progress NeuroPsychopharmacol \& Biol Psychiatry. 2016;69:60-8.

47. Redei EE, Mehta NS. The promise of biomarkers in diagnosing major depression in primary care: the present and future. Curr Psychiatry Rep. 2015;17:601.

48. Zheng P, Chen JJ, Huang T, Wang MJ, Wang Y, Dong MX, et al. A novel urinary metabolite signature for diagnosing major depressive disorder. J Proteome Res. 2013a;12:5904-11.

49. Zheng P, Fang Z, Xu XJ, Liu ML, Du X, Zhang X, et al. Metabolite signature for diagnosing major depressive disorder in peripheral blood mononuclear cells. J Affect Disord. 2016;195:75-81.

50. Zheng $P$, Wang $Y$, Chen L, Yang D, Meng $H$, Zhou D, et al. Identification and validation of urinary metabolite biomarkers for major depressive disorder. Mol \& Cell Proteom. 2013b;12:207-14.

51. Miller DB, O'Callaghan JP. Personalized medicine in major depressive disorder -opportunities and pitfalls. Metab: Clin Exp. 2013;62(Suppl 1):S34-9. 